\title{
Idiopathic Granulomatous Mastitis Associated with Erythema Nodosum
}

\author{
Tuğçe Özlem Kalayc ${ }^{1}$, Melike Bedel Koruyucu ${ }^{1}$, Melda Apaydın ${ }^{1}$, Demet Etit ${ }^{2}$, Makbule Varer ${ }^{1}$
}

${ }^{1}$ Department of Radiology, İzmir Atatürk Training and Research Hospital, İzmir, Turkey

${ }^{2}$ Department of Pathology, İzmir Atatürk Training and Research Hospital, İzmir, Turkey

\section{Background: Idiopathic granulomatous mastitis} (IGM) is an uncommon benign chronic inflammatory breast disease, and erythema nodosum (EN) is an extremely rare systemic manifestation of IGM. Here, we report a rare case of IGM accompanied by EN.

Case Report: A 32-year-old patient was admitted to our clinic with a history of a tender mass in the right breast. On physical examination, the right breast contained a hard, tender mass in the lower half with indrawing of the nipple. She had florid EN affecting both legs. She was evaluated with mammography, ultrasound, power Doppler ultrasound, non-enhancing magnetic resonance imaging (MRI), dynamic contrastenhanced MRI, fine needle aspiration biopsy (FNAB) and excisional biopsy. Time-intensity curves showed a type II pattern on dynamic contrast-enhanced MRI, which has an intermediate probability for malignancy. The FNAB reported a benign cytology suggestive of a granulomatous inflammation, which was also supported by the histopathological findings. A partial mastectomy was performed following medical treatment. There was no recurrence at 1-year follow-up.

Conclusion: IGM should be considered in the differential diagnosis of EN. Although histopathological examination remains the only method for the definite diagnosis of IGM, MRI can be helpful in the diagnosis or differentiation of benign lesions from malignant ones. Keywords: Breast cancer, erythema nodosum, fine needle aspiration biopsy, idiopathic granulomatous mastitis, magnetic resonance imaging, ultrasonography
Idiopathic granulomatous mastitis (IGM) is a rare, benign, chronic inflammatory breast disease first described by Kessler and Wolloch in 1972 (1). IGM is reported to occur predominantly in premenopausal women shortly after their last childbirth. IGM has no confirmed etiology; however, it is associated with breastfeeding, smoking and the use of oral contraceptives $(2,3)$. It is clinically characterized by hard breast lumps with local inflammation, galactorrhea, tumorous indurations and skin ulcerations. Histologically, it is characterized by the presence of non-caseous granulomatous lobulitis $(3,4)$. Erythema nodosum (EN) is an extremely rare systemic manifestation of IGM. It is characterized by red, tender nodules usually located on the extensor surfaces of the legs (5). We present radiologic and histopathological findings of a young woman diagnosed with IGM, which was complicated by EN.

\section{CASE PRESENTATION}

A 32-year-old woman presented with a 1-year history of a tender mass in the right breast. There was no history of local trauma or irritation, breastfeeding, smoking, use of oral contraceptives or any other prescription drugs. Disorders, includ- 


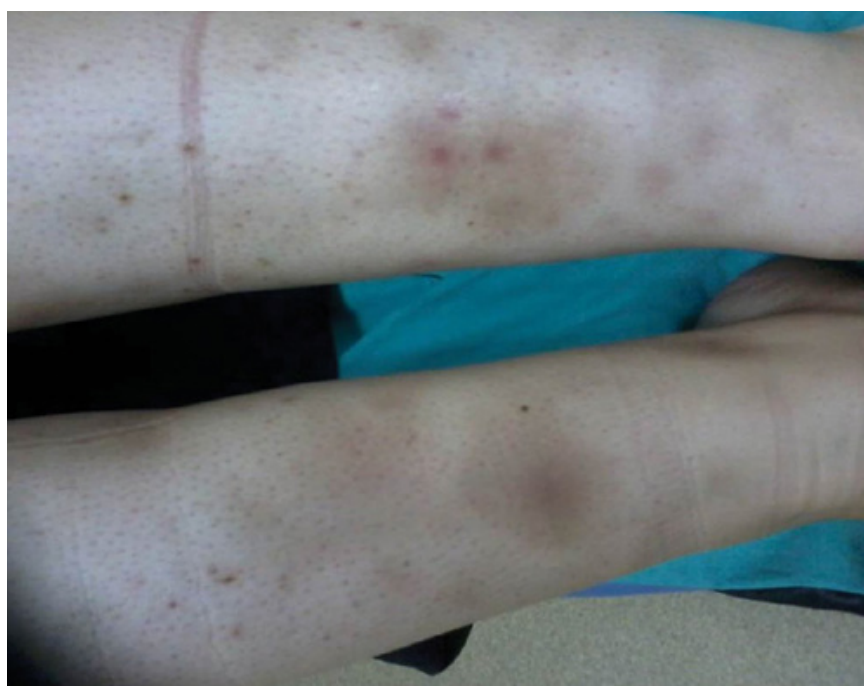

FIG. 1. Erythema nodosum affecting both legs

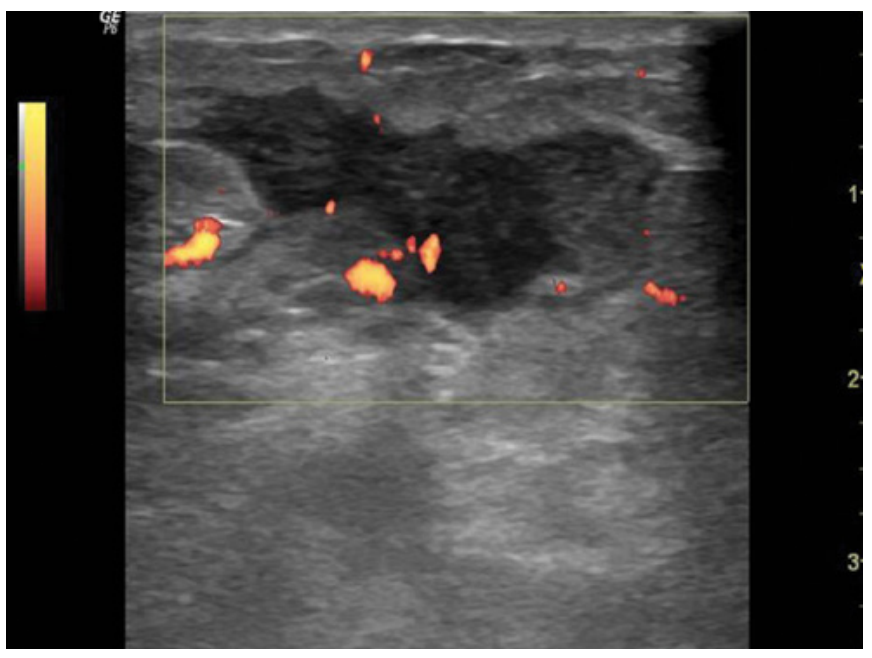

FIG. 2. Ultrasound and power Doppler imaging of lesion demonstrated $3.5 \times 1.2-\mathrm{cm}$ hypoechoic vascular mass with irregular lobulated contour
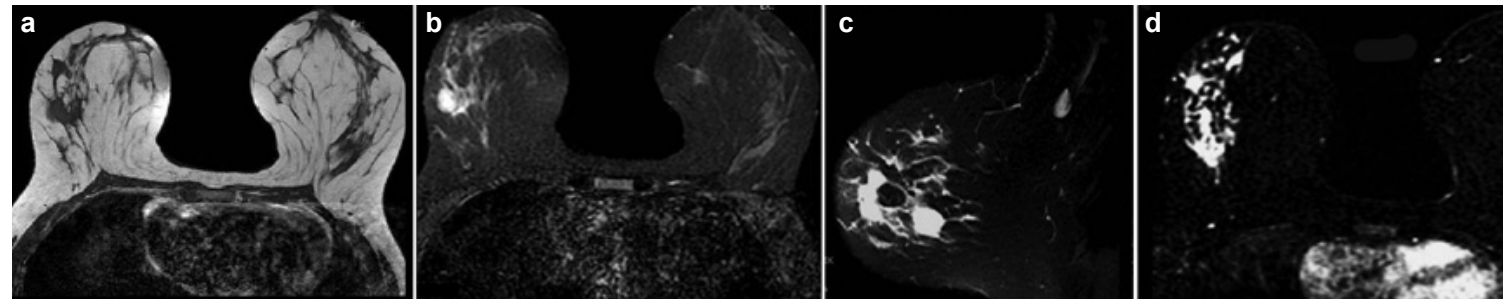

FIG. 3. a-d. Asymmetrical tissue of low signal intensity on the lateral aspect of the right breast is seen on axial pre-contrast T1-weighted (W) image (a), on axial T2-W fat-saturated image, the lesion is seen as hyperintense (b). Sagittal (c) and axial (d) T1-W subtraction images showed heterogeneously enhancing areas with hyperintense nodular enhancements.

ing alpha-1 antitrypsin deficiency and hyperprolactinemia, were not noted. On physical examination, the right breast contained a hard, tender mass in the lower half with in-drawing of the nipple. She had florid EN affecting both legs (Figure 1). Tuberculin skin test and Kveim biopsy results were negative. Wegener's granulomatosis and connective tissue disorders were ruled out by negative test results for auto-antibodies, including proteinase 3 ANCA and ANA. Diabetes mellitus and other systemic disorders were also ruled out in the clinical history and laboratory assessment. Informed consent was obtained from the patient for this case report.

Mammography (MG) was performed in another imaging center; therefore, we had only the written MG report. We refrained from performing another MG to prevent excessive radiation to the young patient. MG reported an irregular, dense right retro-areolar mass. Gray-scale ultrasound (US) and power Doppler US (Toshiba Aplio 500 system; Toshiba Corporation, Tokyo, Japan) showed irregular hypoechoic vascular masses with tubular extensions (Figure 2).

Magnetic resonance imaging (MRI) was performed with a 1.5T Gyroscan Intera system (Philips, Bothell, WA) using a standard protocol that included a T2-weighted (W) rapid spin echo (repetition time msec/echo time msec, 4000/90; section thickness, $4 \mathrm{~mm}$ ) acquisition and 3-dimensional T1-W GRE (20/4.5; flip angle, $30-45^{\circ}$; section thickness, $3 \mathrm{~mm}$ ) acquisition before and after the intravenous administration of contrast material (Gadovist, Bayer Schering Pharma, Berlin, Germany). A bolus injection of $0.1 \mathrm{mmol} / \mathrm{kg}$ was administered, followed by a $10-20 \mathrm{~mL}$ saline flush. Dynamic postcontrast images were obtained within approximately $2 \mathrm{~min}$. On MRI, focal asymmetrical signal intensity changes were seen, which were hypointense on T1-W images and hyperintense on T2-W images with fat saturation and without significant mass effect (Figure 3a, b). Heterogeneously enhancing areas with hyperintense nodular enhancements were seen on dynamic contrast-enhanced MRI (DCE-MRI) (Figure 3c, d). Post-processing of images included subtraction and calculation of time-intensity curves of the enhancing regions. Timeintensity curves showed a type II pattern, which has an intermediate probability for malignancy (Figure 4). The patient did not accept Tru-cut core biopsy; therefore, we performed a fine needle aspiration biopsy (FNAB). FNAB slides showed epithelioid histiocytes and giant cells in a dirty background 

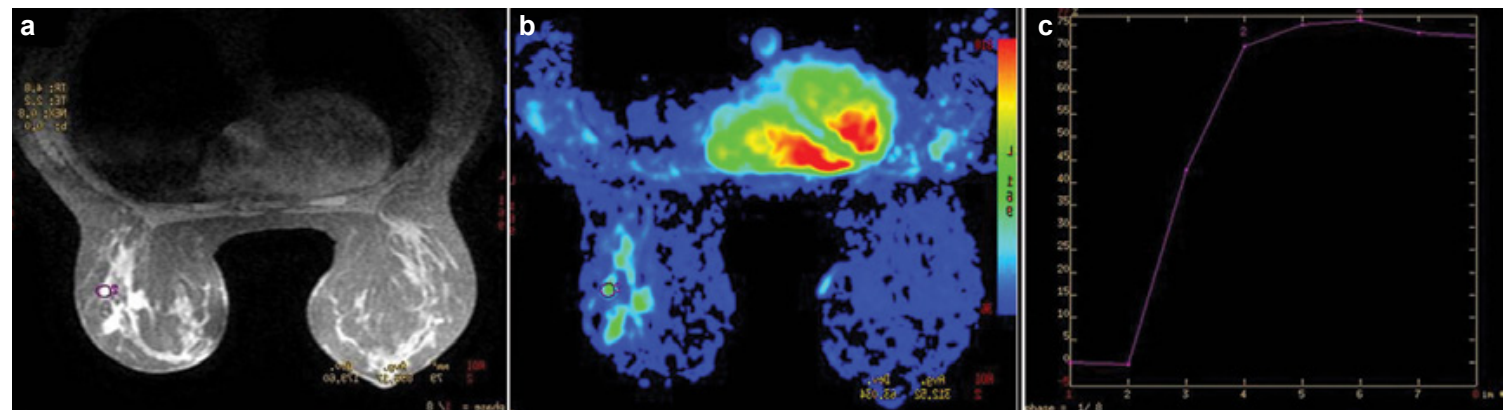

FIG. 4. a-c. Contrast-enhanced T1-weighted, fat-saturated, gradient-echo image shows regional heterogeneous enhancement (a), color-coded map shows foci with the maximum slope of enhancement increase (green) after contrast material injection, the foci were selected as regions of interest (b), dynamic signal intensity time graph (c). Curve indicates plateau enhancement. The vertical axis indicates the percentage of enhancement, and the horizontal axis indicates the time in minutes. A Type II curve has an intermediate probability for malignancy.

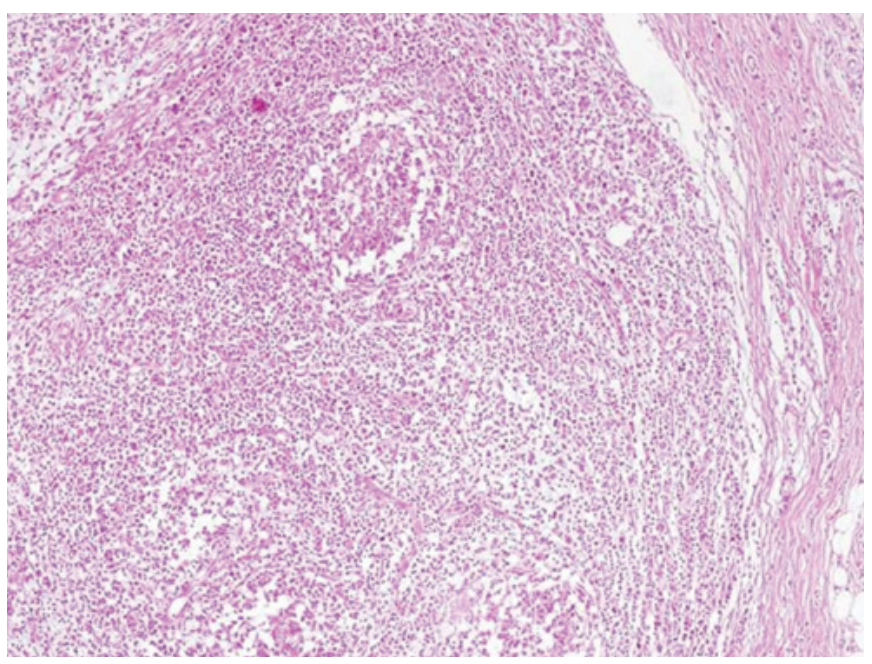

FIG. 5. Photomicrograph of tissue specimen. Note the presence of non-necrotizing granulomatous inflammation with epithelioid histiocytes, lymphocytes, plasma cells, polymorphonuclear leukocytes and multinucleated giant cells (hematoxylin and eosin $\times 100$ ).

supporting a granulomatous inflammation. After the FNAB report, an excisional biopsy was performed. The histopathological findings, including lymphoplasmacytic cell aggregates forming inconspicuous granulomas without necrosis and with microabscesses, supported the granulomatous inflammation (Figure 5). Gram stain, acid-fast bacillus stain, and bacterial, fungal and mycobacterial cultures of the tissue specimen were negative. IGM was a diagnosis of exclusion based on the laboratory tests, histologic staining tests and culture for possible fungal or bacterial causes.

We prescribed oral methylprednisolone $0.8 \mathrm{mg} / \mathrm{kg} /$ day in week 1 with a dose reduction of $0.1 \mathrm{mg} / \mathrm{kg}$ weekly for 8 weeks. At the end of medical treatment, she was evaluated for regression of the breast lesion. Radiologic findings after the completion of therapy showed that the medical treatment was not sufficiently effective on the lesions; therefore, the patient underwent a partial mastectomy. The histopathological diag- nosis after partial mastectomy was the same as the granulomatous mastitis. In the 1-year follow-up period, there was no recurrence according to our medical record database.

\section{DISCUSSION}

Idiopathic granulomatous mastitis is a rare, non-neoplastic disease of uncertain etiology. The incidence of this condition is also uncertain (3). Clinically, the patients present with a breast mass resembling carcinoma, which may be causing nipple retraction and sinus formation. The clinical and radiologic findings of IGM often mimic breast infection or abscess and breast carcinoma $(2,3)$. The diagnosis of IGM is based on the exclusion of other granulomatous diseases, such as tuberculosis, sarcoidosis, Wegener's granulomatosis and connective tissue disorders. In our patient, the negative laboratory results and lack of other clinical features of that spectrum of diseases supported the differential diagnosis $(3,4,6)$.

Erythema nodosum (EN) is a panniculitis which may have various causes, such as sarcoidosis, tuberculosis, infections or drugs, or it can be idiopathic (5). It is a rare systemic manifestation of IGM $(3,6)$. Adams et al. (3) reported the first case of IGM complicated by EN in 1987. Although there are many case reports of granulomatous mastitis associated with EN, only a few cases have reported IGM associated with EN (6). Adams et al. (3) reported that EN might be supportive evidence of an autoimmune component in the etiology of IGM.

Mammography and US are generally used for the diagnosis of IGM. The most common presentation in MG is a large, focal asymmetric density or a lobulated, irregular mass that does not cause mass effect, as well as multiple small, ill-defined masses and diffusely increased density. The most common finding in US is a large, irregular hypoechoic mass with multiple tubular extensions, lobulated irregular hypoechoic mass or parenchymal distortion with acoustic shadowing and no 
discrete mass. In our case, US appeared as an irregular hypoechoic mass with multiple tubular extensions. The findings of US were consistent with previously reported cases of IGM in the literature $(2,7)$. The radiological spectrum of findings in US is wide. Due to this wide spectrum and low sensitivity, the findings of MG and US are challenging. Therefore, these methods are mainly used to rule out malignancy rather than to confirm the diagnosis. On MRI, IGM usually demonstrates focal or diffuse asymmetrical signal intensity and enhancement or it may demonstrate irregular or spiculated contours, which may lead to misdiagnosis $(2,3,7)$.

Dynamic contrast-enhanced MRI of the breast has been proposed to increase the specificity of the diagnosis of IGM (2).

Specific patterns of enhancement have been classified as persistent (type I), plateau (type II) and washout (type III). The Type I or persistently enhancing (progressive) pattern is suggestive of a benign lesion. The Type II or plateau-type pattern has an intermediate probability for malignancy. The Type III or washout-type pattern is strongly associated with malignancy $(2,8,9)$. The few studies using DCE-MRI to evaluate IGM have revealed a variety of radiological appearances and enhancement patterns, including ring-like areas of enhancement in some patients, areas of nodular enhancement, and heterogeneously enhanced areas without mass effect $(8,9)$. DCEMRI with time-signal intensity curves plays an important role in differentiating IGM from malignancy if a benign type I signal intensity time curve is obtained. In some cases, type II or malignant type III curves can be obtained (2,7-9). In our case, differentiation from malignancy by DCE-MRI was difficult, because time-intensity curves showed a type II pattern, which is seen in both benign and malignant lesions.

The patient's cytology report identified a benign inflammatory process, a granulomatous inflammation, which was supported on the histopathological sections. Chronic granulomatous inflammation with giant cells, neutrophils, epithelioid cells and foamy macrophages was also seen in an abscess formation on the cut sections of the excisional biopsy.

In conclusion, the coincidence of IGM and EN is rare. The imaging findings of IGM overlap with those of malignancy. MRI can be helpful in the diagnosis of disease or in the differentiation of benign lesions from malignant ones. Imaging modalities are important for guidance, but they are not sufficient alone. Knowledge of patient history and essential clinical and laboratory findings are also needed. Core needle biopsy or excisional biopsy procedures are mandatory for a definitive diagnosis.

Ethics Committee Approval: Ethics committee approval was received for this study from the The Institutional Ethics Committee.
Informed Consent: Written informed consent was obtained from patient who participated in this case.

Peer-review: Externally peer-reviewed.

Author contributions: Concept - T.Ö.K., M.B.K., M.A.; Design T.Ö.K., M.B.K., M.A.; Supervision - T.Ö.K., M.B.K., M.V.; Resource - T.Ö.K.; Materials T.Ö.K., M.B.K., M.A., D.E.; Data Collection \&/ or Processing - T.Ö.K.; Analysis \&/or Interpretation - T.Ö.K., D.E., M.V.; Literature Search - T.Ö.K., D.E., M.V.; Writing - T.Ö.K.; Critical Reviews - T.Ö.K., M.B.K., M.A., D.E., M.V.

Conflict of Interest: No conflict of interest was declared by the authors.

Financial Disclosure: The authors declared that this study has received no financial support.

\section{REFERENCES}

1. Kessler E, Wolloch Y. Granulomatous mastitis: A lesion clinically simulating carcinoma. Am J Clin Pathol 1972;58:642-6. [CrossRef]

2. Anik Y. Radiological findings of idiopathic granulomatous mastitis: Review. Turkiye Klinikleri J Med Sci 2010;30:286-92. [CrossRef]

3. Adams DH, Hubscher SG, Scott DG. Granulomatous mastitis - a rare cause of erythema nodosum. Postgrad Med J 1987;63:581-2. [CrossRef]

4. Vinayagam R, Cox J, Webb L. Granulomatous mastitis: A spectrum of disease. Breast Care (Basel) 2009;4:251-4. [CrossRef]

5. Koufakis T, Gabranis I. Erythema nodosum leads to the diagnosis of pulmonary tuberculosis. Pan Afr Med J 2014;18:291. [CrossRef]

6. Nakamura T, Yoshioka K, Miyashita T, Ikeda K, Ogawa Y, Inoue $\mathrm{T}$, et al Granulomatous mastitis complicated by arthralgia and erythema nodosum successfully treated with prednisolone and methotrexate. Intern Med 2012;51:2957-60. [CrossRef]

7. Larsen LJH, Peyvandi B, Klipfel N, Grant E, Iyengar G. Granulomatous lobular mastitis: Imaging, diagnosis, and treatment. AJR Am J Roentgenol 2009;193:574-81. [CrossRef]

8. El Khouli RH, Macura KJ, Jacobs MA, Khalil TH, Kamel IR, Dwyer A, et al. Dynamic contrast-enhanced MRI of the breast: Quantitative method for kinetic curve type assessment. AJR Am J Roentgenol 2009;193:295-300. [CrossRef]

9. Kocaoglu M, Somuncu I, Ors F, Bulakbası N, Tayfun C, Ilkbahar S. Imaging findings in idiopathic granulomatous mastitis. A review with emphasis on magnetic resonance imaging. $J$ Comput Assist Tomogr 2004;28:635-41. [CrossRef] 